\title{
Relación de Capacitación con Adopción de Internet y E- Commerce: Diferencias entre Microemprendedores de Chile
}

\author{
Gustavo A. Barrera \\ Universidad Tecnológica de Chile INACAP, Dirección de Postgrados. Avenida del Valle Norte 819, \\ Huechuraba, Santiago- Chile. (e-mail: gbarrera@inacap.cl)
}

Recibido Abr. 25, 2017; Aceptado Jun. 27, 2017; Versión final Jul. 24, 2017, Publicado Dic. 2017

\begin{abstract}
Resumen
La investigación que se presenta busca relacionar capacitación en micro-emprendedores en Chile con adopción de Internet y e-Commerce en su organización. Adicionalmente, estudiar la incidencia de edad, género, nivel educacional del emprendedor, número de socios de la organización y fuente de financiamiento de la capacitación, en adopción de Internet y e-Commerce, en grupo de micro-emprendedores capacitados. El análisis se efectúa a través de test de hipótesis y regresiones logísticas. Los resultados muestran relación entre capacitación y adopción de Internet y actividades e-Commerce seleccionadas, exceptuando la venta de productos. También indican que emprendedores con menor edad, género femenino, y mayor nivel educacional, adoptan Internet en sus empresas y efectúan algunas actividades e-Commerce en mayor medida. Por último, se evidencia que en microempresas con mayor cantidad de socios y que financian capacitación con recursos propios, se utiliza en mayor proporción Internet y algunas actividades eCommerce seleccionadas.
\end{abstract}

Palabras claves: microemprendimiento; internet; e-commerce; capacitation; características demográficas.

\section{Relationship of Training with Internet Adoption and E- Commerce: Differences in Microentrepreneurs from Chile}

\begin{abstract}
The research seeks to relate training in micro-entrepreneurs in Chile with Internet and e-Commerce adoption in their organization. Additionally, it studies the incidence of age, gender, educational level of the entrepreneur, number of partners of the organization and source of funding for training, in Internet adoption and e-Commerce in a group of micro-entrepreneurs trained. The analysis is performed through hypothesis test and logistic regressions. Results show the relationship between training and Internet adoption and selected e-Commerce activities, except for the sale of products. They also indicate that younger entrepreneurs, of female gender, and higher educational level, adopt the Internet in their companies and carry out some e-Commerce activities to a greater extent. Finally, it is shown that in microenterprises with more partners and that finance training with their own resources, Internet and some selected e-Commerce activities, are used in greater proportion.
\end{abstract}

Keywords: micro entrepreneurship; internet; e-commerce; training; demographic characteristics. 


\section{INTRODUCCIÓN}

El desarrollo de Internet ha transformado los negocios en los últimos años, cada vez más organizaciones obtienen acceso a clientes y proveedores ubicados en diferentes ciudades y países. Se ha reconocido que la incorporación de TICs en las empresas apoyado el desarrollo de negocios globales (lansiti y Lakhani, 2014) también que mejora la productividad de las empresas (Colombo et al., 2013). En pequeñas y microempresas, la utilización de Internet y el desarrollo de e-Commerce es una oportunidad, pues les permite incrementar su capacidad para difundir productos, realizar ventas a distancia y flexibilizar su estructura de costos. En países latinoamericanos emergentes el uso de internet es significativo, lo que implica oportunidades de negocio para microempresas, ejemplo de ello, es que Chile lidera en e-Commerce en la región (CCS, 2016) y $72,4 \%$ de su población presentaba acceso a Internet en 2015 (Subtel, 2016). En forma complementaria, diversos autores han reconocido barreras que dificultan la adopción de e-Commerce en pequeñas y medianas empresa, dentro de estas barreras, la falta de formación y conocimientos es identificada como una dificultad relevante para incorporar e-Commerce en organizaciones (Lawson et al., 2003).

De igual forma, se han estimado beneficios de la formación de emprendedores sobre desempeño de sus organizaciones, específicamente, se ha relacionado en forma positiva la participación en capacitación y el desempeño emprendedor (Martin et al., 2013). A pesar del reconocimiento de beneficios del entrenamiento en emprendedores, no se ha vinculado con adopción de Internet y e-Commerce en microempresas de Latinoamérica, considerando e-Commerce, en un sentido amplio, como la venta de productos, la promoción y publicidad de productos, prestación de servicios de apoyo y desarrollo de alianzas a través de Internet (Turban et al., 2015); este conocimiento es especialmente relevante en países latinoamericanos emergentes como Chile, Colombia, México y Brasil (MSCI World Index Stock, 2016), pues en ellos, las microempresas representan una alta participación en oferta de puestos de trabajo y existe importante interés en apoyar su desarrollo (Orjuela, 2006), adicionalmente, se ha reconocido baja adopción de Internet en emprendimientos chilenos (GEM, 2015).

En consecuencia, la investigación presentada busca asociar la participación de micro-emprendedores en capacitación con adopción de Internet y actividades de e-Commerce seleccionadas, diferenciando su vínculo por condiciones del emprendedor y su empresa; se busca extender el conocimiento asociado a adopción de Internet y e-Commerce hacia microempresas latinoamericanas, pues mayoritariamente, las investigaciones en este ámbito han estudiado pequeñas y medianas empresas de países de Europa, EEEUU, Asia y África. Una mayor comprensión de la relación de capacitación con adopción de Internet y e-Commerce, bajo condiciones específicas del emprendedor y su organización, facilitaría el desarrollo de programas de capacitación adaptados a las características del micro-emprendedor para incrementar su efectividad (Haider et al., 2015; Nasri y Iskandar, 2015).

\section{MARCO TEÓRICO}

Se ha estimado que no es factible para las organizaciones mantenerse al margen de los avances de tecnologías de información y comunicación, debido al creciente acceso a Internet e nivel global (Vidal et al, 2017), también que el uso de estas tecnologías las beneficia en diversos ámbitos, como acceso a información de nuevos mercados, reducción de costos de transacción, mayor acceso a mercados y apoyo para efectuar contactos con clientes (Qureshi, 2005; Kotelnikov, 2007). En el contexto de microempresas se ha reconocido que las TICs facilitan la comprensión de los consumidores y la obtención de ventajas competitivas (Cataldo y McQueen, 2014), y que las empresas pequeñas son más susceptibles a percibir perjuicios por el desarrollo tecnológico, al presentar mayor dificultad en la adopción de nuevas tecnologías (Ollivier Fierro, 2007).

En relación con los beneficios del e-Commerce para las empresas, se han destacado diversas ventajas, como mejoras en productividad a través de automatización de las transacciones (Gallego, 2016) y la consolidación de oferta y demanda por medio de intermediarios digitales (Lin, 2014). En el ámbito de pequeñas y medianas empresas, también se reconocen beneficios, Julta, et al. (2002) estiman que el e-Commerce permite a las pequeñas empresas competir con grandes organizaciones y suplir sus deficiencias de conocimientos y experiencia, Afshar et al. (2013) plantean que el e-Commerce es necesario en estas empresas para abordar oportunidades de ventas a nivel internacional.

Respecto del estudio de dificultades en la adopción de TICs en microempresas, Chong (2013) afirma que las pequeñas empresas perciben mayores barreras al implementar tecnologías de información pues las consideran costosas, riesgosas y complejas, por su parte, Wolcott et al. (2008) afirman que existen mayores desafíos en la adopción de TICs en microempresas, pues su personal presenta bajo entrenamiento y desconocen sus beneficios. En el ámbito específico de e-Commerce, también se han identificado aspectos relevantes que afectan su adopción, como la actitud de la dirección de empresa frente al e-Commerce (Simmons et al., 2008) y conocimientos y actitudes del personal que inciden en su implementación (Bruque y 
Moyano, 2007); en Latinoamérica, los estudios efectuados siguen sentido similar, por ejemplo, Nasco et al. (2008) estiman que las actitudes de jefaturas de empresas chilenas inciden en su intención de adoptar eCommerce en las organizaciones que dirigen. Las investigaciones mencionadas reconocen que conocimientos y actitudes de directivos de empresas afectan la adopción de tecnología y e-Commerce en sus organizaciones, en coherencia con esta evidencia, se estima que la participación de microemprendedores en programas de formación, se relaciona en forma positiva con uso de Internet y desarrollo de actividades eCommerce en sus empresas.

La educación para el emprendimiento es definida como la intervención de un educador para desarrollar, aprendizaje en temáticas relacionadas con emprendimiento o habilidades para subsistir en el ámbito de los negocios (Valerio et al., 2014), también, como el desarrollo formal y estructurado de competencias empresariales, a través de la transferencia de conceptos y el fortalecimiento de habilidades individuales, utilizadas al inicio o en el desarrollo de empresas (Alberti et al., 2004). Se han reconocido diversos beneficios de la educación y programas de entrenamiento sobre emprendedores, se considera que crean apertura y confianza para el emprendimiento (Elmuti et al., 2012), que inciden en la actitud emprendedora y en la intención de crear nuevas empresas (Martin et al., 2013), que beneficia las habilidades de gestión (Putta, 2014), y que ayudan al desarrollo ventas y utilidades de pequeñas y medianas empresas (Njoroge y Gathungu, 2013); en el ámbito de microempresas, el entrenamiento se ha vinculado con incremento de ingresos (Kessy y Temu, 2010) y logro de objetivos empresariales (Dumas, 2001).

Respecto de resultados de la capacitación por género, Rauth (2014) demuestra que el entrenamiento ayuda a las mujeres a encontrar financiamiento para desarrollar sus empresas, Petridou et al. (2009) plantean que las mujeres muestran mayor interés que los hombres en adquirir conocimientos y capacidades, y Do Paço et al. (2015), estiman que las mujeres que participan en escuelas de negocio de educación superior presentan mayor intención de emprender dentro de su género. En relación con diferencia en resultados de empresarios capacitados por su edad, Ramírez-Pérez et al. (2015) muestran que emprendedores mayores exhiben mayores habilidades de comunicación y organización, Martin et al. (2013) evidencian que la educación en emprendimiento es más efectiva en el desarrollo de actitudes en emprendedores jóvenes, y Ashourizadeh et al. (2014) plantean que existen menores resultados en entrenamiento de emprendedores de generaciones mayores.

En al ámbito de estudio sobre incidencia de nivel de educación formal en emprendimiento, se ha reconocido que favorece la intención de emprender (Bae et al. 2014), que existe vínculo de educación terciaria y emprendimiento de población en EEUU (Millan et al., 2014), también que un mayor nivel educacional incrementa las capacidades técnicas y habilidades del emprendedor (Gomezelj y Antončič, 2008), y que la educación formal previa, favorece los resultados del entrenamiento de emprendedores (Ashourizadeh et al., 2014). Respecto del tamaño de empresa, Kotey y Folker (2007) muestran que, en contexto de Pymes, las empresas más pequeñas, presenta menor propensión a la capacitación formal y que emprendimientos de menor tamaño se orientan hacia el aprendizaje informal.

Las investigaciones presentadas reconocen los beneficios de la capacitación en empresas y la incidencia de condiciones edad, género, nivel educacional y tamaño de la empresa, en países con culturas y economías diferentes, como Egipto e Irán (Ashourizadeh et al., 2014), Malasya (Haider et al., 2015), Tanzania (Kessy, y Temu, 2010), Australia (Kotey y Folker, 2007), EE. UU. (Millan et al., 2014) e India (Rauth, 2014). Se estima que existe brecha de conocimiento de esta temática en Chile y Latinoamérica, y que no se ha estudiado con mayor profundidad, las implicancias de número de socios y fuente de financiamiento sobre resultados de capacitación.

\section{OBJETIVOS DE INVESTIGACIÓN}

El objetivo de la investigación es relacionar participación en capacitación con adopción de internet y actividades e-Commerce en empresas de Chile, específicamente:

(i) Evaluar diferencias en adopción de Internet; promoción de productos, relacionamiento con proveedores y clientes, y venta de productos por Internet, entre micro-emprendedores que participan y no participan en capacitación.

(ii) Analizar variación de adopción de Internet y actividades e-Commerce, por edad, género y nivel educacional de micro-emprendedores capacitados.

(iii) Estimar diferencias de adopción de internet y actividades e-Commerce entre emprendedores capacitados, en función de número de socios de su empresa y fuente de financiamiento para capacitación. 


\section{METODOLOGÍA}

Para comprobar las asociaciones propuestas, son utilizadas las respuestas obtenidas por la Cuarta Encuesta de Emprendimiento, efectuada por el Ministerio de Economía Fomento y Turismo De Chile (2016), este estudio, incorpora respuestas de 7.543 microempresas diferenciadas por tamaño y tipo de industria, son consideradas como microempresas, las organizaciones con menos de 10 trabajadores. Desde esta muestra son seleccionadas 6.135 empresas que declararon mantener sus actividades emprendedoras en 2015 , de estas empresas 1.356 micro-emprendedores confirmaron su participación en alguna capacitación durante los últimos 5 años. En tablas 1 y 2, son presentadas las consultas seleccionadas de encuesta, para identificar condiciones de micro-emprendedores y de sus organizaciones, y su adopción de Internet y actividades eCommerce.

Tabla 1: Preguntas en encuesta para evaluar adopción de Internet, e-Commerce y capacitación. Tomada de: Cuarta Encuesta de Emprendimiento Ministerio de Economía de Chile (2016)

\begin{tabular}{|l|l|l|}
\hline \multicolumn{1}{|c|}{ Variable } & \multicolumn{1}{c|}{ Pregunta de encuesta } & \multicolumn{1}{c|}{$\begin{array}{c}\text { Alternativas de } \\
\text { respuesta }\end{array}$} \\
\hline $\begin{array}{l}\text { Utiliza Internet en la } \\
\text { empresa }\end{array}$ & Para su negocio ¿usted utiliza internet? & $1=$ Sí,0=No \\
\hline $\begin{array}{l}\text { Efectúa Promoción de } \\
\text { productos, relación con } \\
\text { clientes y proveedores, o } \\
\text { venta de a través de } \\
\text { Internet }\end{array}$ & $\begin{array}{l}\text { ¿Qué usos le da a internet en su empresa?: Promocionar su } \\
\text { empresa, racionamiento con clientes y proveedores, venta de } \\
\text { productos. }\end{array}$ & $1=$ Sí,0=No \\
\hline $\begin{array}{l}\text { Participación en } \\
\text { capacitación }\end{array}$ & $\begin{array}{l}\text { ¿Ha recibido algún tipo de capacitación para la actividad } \\
\text { económica que realiza durante los últimos 5 años? }\end{array}$ & $1=$ Sí,0=No \\
\hline
\end{tabular}

Tabla 2: Preguntas en encuesta para evaluar condiciones del micro-emprendedor y su organización. Tomada de: Fuente: Cuarta Encuesta de Emprendimiento Ministerio de Economía de Chile (2016)

\begin{tabular}{|l|l|}
\hline \multicolumn{1}{|c|}{ Variable } & \multicolumn{1}{c|}{ Alternativas de respuesta } \\
\hline Edad & Respuesta abierta \\
\hline Género & 1= Hombre, 0=Mujer \\
\hline Nivel educacional & $\begin{array}{l}\text { 0=Nunca asistió, 1=Sala Cuna/Jardín Infantil, 2= Kinder/Pre-kinder, 3=Básica 0 } \\
\text { Primaria, 4=Media Común/Media Técnico Profesional/ Humanidades, 5=Centro } \\
\text { de Formación Técnica, 6=Instituto Profesional, 7=Universitario, 8=Postítulo, } \\
\text { 9=Magíster, 10=Doctorado }\end{array}$ \\
\hline Fuente de financiamiento & $\begin{array}{l}\text { 1=Recursos de la empresa o negocio, 0=Externo: Programas financiados con } \\
\text { fondos públicos y/o privados }\end{array}$ \\
\hline Número de socios en la empresa & Respuesta abierta \\
\hline
\end{tabular}

La metodología utilizada para efectuar análisis es, en primer lugar, pruebas de hipótesis a través de test con estadígrafo Chi2 para evaluar diferencias de emprendedores capacitados y no capacitados respecto de adopción de Internet y actividades e-Commerce seleccionadas (considerando clasificación ampliada de Turban et al., 2015); los resultados son diferenciados por género, pues el grupo de micro-emprendedores capacitado presenta mayor proporción de mujeres que el grupo que no participa en capacitación (Test Chi2; $\operatorname{Pr}=0,000)$. En segundo lugar, se efectúan regresiones logísticas para corroborar la incidencia de características del emprendedor y su organización en adopción de Internet y actividades e-Commerce, se analizan resultados sólo en el grupo que participa en capacitación. Las regresiones logísticas planteadas, son las siguientes:

Adopción de Internet $=\beta 0+\beta 1 *$ Edad $+\beta 2 *$ Sexo $+\beta 3 *$ Nivel Educacional $+\beta 4 *$

Fuente de Financiamiento $+\beta 5 *$ Número de socios

Promomción de productos $=\beta 0+\beta 1 *$ Edad $+\beta 2 *$ Sexo $+\beta 3 *$ Nivel Educacional $+\beta 4 *$

Fuente de Financiamiento $+\beta 5 *$ Número de socios

Relación con clientes y proveedores $=\beta 0+\beta 1 *$ Edad $+\beta 2 *$ Sexo $+\beta 3 *$ Nivel Educacional +

$\beta 4 *$ Fuente de Financiamiento $+\beta 5 *$ Número de socios

Venta de productos $=\beta 0+\beta 1 *$ Edad $+\beta 2 *$ Sexo $+\beta 3 *$ Nivel Educacional $+\beta 4 *$

Fuente de Financiamiento $+\beta 5 *$ Número de socios 


\section{RESULTADOS Y DISCUSION}

Los resultados del análisis Chi2, que estima diferencias en micro-emprendedores capacitados respecto de uso de Internet y actividades e-Commerce, muestran que existen diferencias en la adopción de internet $\operatorname{Pr}$ Chi $2=0,00$ ), en la promoción de productos ( $\operatorname{Pr}$ Chi $2=0,02$ ) y en relacionamiento con clientes y proveedores (Pr Chi 2=0,03), entre los grupos emprendedores capacitados y no capacitados totales, sólo la venta de productos a través de internet, no presenta diferencias entre estos grupos ( $\operatorname{Pr} C h i 2=0,02)$. Se evidencia que los micro-emprendedores capacitados adoptan Internet, promocionan su empresa y se relacionan con clientes y proveedores a través Internet en mayor proporción. No se evidencian diferencias de resultados significativas, por género.

Respecto de diferencias entre grupos de emprendedores capacitados por temáticas, se reconocen diferencias en uso de Internet ( $\operatorname{Pr}$ Chi $2=0,01$ ) y promoción de productos ( $\operatorname{Pr}$ Chi $2=0,01$ ), se evidencia que los emprendedores que han participado en capacitación relacionadas con temas de gestión, comercialización, ventas, tecnologías de información, innovación, presentan mayor propensión al uso de Internet y promoción de productos. Adicionalmente, no se reconocer diferencias para venta de productos y relacionamiento con clientes y proveedores, a través de Internet. Al dividir el análisis por género, se obtienen resultados similares, la diferencia más relevante se encuentra al comparar microempresarios capacitados en administración, marketing y ventas, innovación y TICs con otros temas, pues se reconoce que sólo los hombres capacitados en estas temáticas presentan mayor adopción de Internet $(\mathrm{Chi} 2=0,00)$ y sólo las mujeres capacitados en estas temáticas muestran mayor adopción de promoción de productos a través de Internet $(C h i 2=0,01)$. La tabla 3, describe los resultados.

Tabla 3: Test de hipótesis relación de capacitación con adopción de Internet y e-Commerce. *** Coeficiente significativo con $99 \%$ de confianza, ${ }^{* *}$ Coeficiente significativo con $95 \%$ de confianza, ${ }^{*}$ Coeficiente significativa con $90 \%$ de confianza.

\begin{tabular}{|c|c|c|c|c|c|c|c|c|c|}
\hline & $\begin{array}{l}\text { Se ha } \\
\text { capacitado }\end{array}$ & $\begin{array}{l}\text { Utiliza } \\
\text { Internet } \\
\text { (Sí-No) }\end{array}$ & $\begin{array}{l}\text { Pr } \\
\text { Chi2 }\end{array}$ & $\begin{array}{l}\text { Promociona } \\
\text { productos } \\
\text { (Sí-No) }\end{array}$ & $\begin{array}{l}\text { Pr } \\
\text { Chi2 }\end{array}$ & $\begin{array}{l}\text { Vende } \\
\text { productos } \\
\text { (Sí-No) }\end{array}$ & $\begin{array}{l}\text { Pr } \\
\text { Chi2 }\end{array}$ & $\begin{array}{l}\text { Se relaciona } \\
\text { con clientes } \\
\text { proveedores } \\
\text { (Sí-No) }\end{array}$ & $\begin{array}{l}\text { Pr } \\
\text { Chi2 }\end{array}$ \\
\hline \multirow{2}{*}{$\begin{array}{l}\bar{\pi} \\
\stackrel{0}{0}\end{array}$} & Sí & $741-615$ & \multirow{2}{*}{$\underset{* * *}{0,00}$} & $420-321$ & \multirow{2}{*}{$\underset{* *}{0,02}$} & $310-431$ & \multirow{2}{*}{0,16} & $619-122$ & \multirow{2}{*}{$\underset{\star \star}{0,03}$} \\
\hline & No & $1.449-3.327$ & & $743-706$ & & $561-888$ & & $1156-293$ & \\
\hline \multirow{2}{*}{$\begin{array}{l}\text { 이 } \\
\text { 흘 } \\
\text { 우 }\end{array}$} & Sí & $397-317$ & \multirow{2}{*}{$\underset{* * *}{0,00}$} & $209-188$ & \multirow{2}{*}{$\underset{*}{0,08}$} & $164-233$ & \multirow{2}{*}{0,33} & $338-59$ & \multirow{2}{*}{$\underset{*}{0,08}$} \\
\hline & No & $1.006-2.161$ & & $478-528$ & & $387-619$ & & $817-189$ & \\
\hline \multirow{3}{*}{$\frac{\frac{1}{\sigma}}{\frac{1}{\Sigma}}$} & Sí & $344-298$ & \multirow{2}{*}{$\begin{array}{c}* * * \\
0,00\end{array}$} & $211-133$ & \multirow{2}{*}{0,67} & $146-198$ & \multirow{2}{*}{0,37} & $281-63$ & \multirow{2}{*}{$\underset{*}{0,08}$} \\
\hline & No & $443-1.166$ & & $265-178$ & & $174-269$ & & $339-104$ & \\
\hline & $\begin{array}{l}\text { Capacitación } \\
\text { en temas } \\
\text { seleccionados }\end{array}$ & $\begin{array}{l}\text { Utiliza } \\
\text { Internet } \\
\text { (Sí-No) }\end{array}$ & $\begin{array}{l}\mathrm{Pr} \\
\text { Chi2 }\end{array}$ & $\begin{array}{l}\text { Promociona } \\
\text { productos } \\
\text { (Sí-No) }\end{array}$ & $\begin{array}{l}\text { Pr } \\
\text { Chi2 }\end{array}$ & $\begin{array}{l}\text { Vende } \\
\text { productos } \\
\text { (Sí-No) }\end{array}$ & $\begin{array}{l}\mathrm{Pr} \\
\text { Chi2 }\end{array}$ & $\begin{array}{l}\text { Se relaciona } \\
\text { con clientes } \\
\text { proveedores } \\
\text { (Sí-No) }\end{array}$ & $\begin{array}{l}\mathrm{Pr} \\
\text { Chi2 }\end{array}$ \\
\hline \multirow{2}{*}{$\begin{array}{l}\bar{\pi} \\
\frac{\sigma}{0}\end{array}$} & Sí & $215-137$ & \multirow{2}{*}{$\underset{* *}{0,01}$} & $139-76$ & \multirow{2}{*}{$\underset{* *}{0,01}$} & $97-188$ & \multirow{2}{*}{0,27} & $178-47$ & \multirow{2}{*}{0,67} \\
\hline & No & $471-521$ & & $278-243$ & & 212-309 & & $438-83$ & \\
\hline \multirow{2}{*}{$\begin{array}{l}\text { 흐 } \\
\text { 트 } \\
\text { 오 }\end{array}$} & Sí & $108-40$ & \multirow{2}{*}{$\underset{* * *}{0,00}$} & $62-46$ & \multirow{2}{*}{0,25} & $50-58$ & \multirow{2}{*}{0,23} & $93-15$ & \multirow{2}{*}{0,83} \\
\hline & No & $285-275$ & & $145-140$ & & $113-172$ & & $243-42$ & \\
\hline \multirow{2}{*}{$\frac{\frac{1}{2}}{\frac{1}{2}}$} & Sí & $107-97$ & \multirow{2}{*}{0,61} & $77-30$ & \multirow{2}{*}{0,01} & $47-60$ & \multirow{2}{*}{0,73} & $85-22$ & \multirow{2}{*}{0,48} \\
\hline & No & 236-196 & & $133-103$ & & $99-137$ & & $196-41$ & \\
\hline
\end{tabular}

También se aprecia que los micro-emprendedores capacitados durante los últimos cinco años ( $\mathrm{n}=1.356)$ presentan media aritmética de edad menor (49 años) que aquellos que no han participado de capacitación (51 años; ANOVA con Prob>chi2=0,000), también, que los micro-emprendedores que se capacitan tienden a disponer de mayor nivel de educación previo (ANOVA con Prob>chi2 $=0,000$ ), en consecuencia, es posible plantear que si bien existen diferencias entre grupos, estas podrían derivarse de diferencias de edad y formación de sus integrantes.

Respecto de relación de características del emprendedor, el número de socios de la empresa y la fuente de financiamiento de capacitación, con adopción de Internet y actividades e-Commerce en el grupo total de micro-emprendedores capacitados (casos válidos $=1205$ y 630), los resultados de regresiones logísticas muestran que la mayor edad de los emprendedores se relaciona con menor adopción de Internet (Odds ratio 
$0,968, p>z=0,000$ ), menor promoción de empresa (Odds ratio $0,957, p>z=0,000$ ), menor relacionamiento con clientes y proveedores (Odds ratio $0,983, p>z=0,048$ ), y menor venta de productos a través de Internet (Odds ratio $0,980, p>z=0,002)$; el género femenino se relaciona en forma favorable con mayor promoción de productos a través de Internet (Odds ratio $0,725, p>z=0,069$ ); el nivel de estudios se asocia en forma positiva con mayor adopción de Internet (Odds ratio $2,289, p>z=0,000$ ) y mayor relación con clientes y proveedores a través de Internet (Odds ratio 1,267, $p>z=0,003$ ); el financiamiento de capacitación con recursos de la empresa se vincula en forma positiva con mayor utilización de Internet (Odds ratio $0,439, p>z=0,000$ ) y mayor relación con clientes y proveedores (Odds ratio $0,541, p>z=0,016$ ) y una mayor cantidad de socios en la empresa se asocia con mayor utilización de Internet (Odds ratio 1,511, $p>z=0,003$ ).

Al estudiar la variables señaladas, con la adopción de Internet y actividades e-Commerce, en el grupo de micro-emprendedores capacitados en gestión, marketing y ventas, innovación y TICs (casos válidos=326 y 197), los resultados evidencian que la mayor edad de los emprendedores se relaciona con menor adopción de Internet (Odds ratio 0,949, $p>z=0,000$ ); el género femenino se vincula en forma favorable con mayor promoción de productos a través de Internet (Odds ratio $0,421, p>z=0,019$ ); el nivel de estudios se asocia en forma positiva con mayor adopción de Internet (Odds ratio $1,754, p>z=0,000$ ) y mayor promoción de empresa a través de Internet (Odds ratio 1,289, $p>z=0,032$ ); el financiamiento de capacitación con recursos de la empresa se relaciona en forma positiva con mayor utilización de Internet (Odds ratio $0,228, p>z=0,001$ ) y mayor relación con clientes y proveedores (Odds ratio $0,402, p>z=0,082$ ) y una mayor cantidad de socios en la empresa se vincula con mayor utilización de Internet (Odds ratio 2,331, $p>z=0,016$ ) y mayor relación con clientes y proveedores (Odds ratio $3,740, p>z=0,030$ ). Las Tablas 4 y 5 presentan los resultados de regresiones.

Tabla 4: Probabilidad de adopción en micro-emprendedores capacitados totales. ${ }^{* * \star}$ Coeficiente significativo, 99\% de confianza. ${ }^{* *}$ Coeficiente significativo, $95 \%$ de confianza. ${ }^{*}$ Coeficiente significativo, $90 \%$ de confianza.

\begin{tabular}{|c|c|c|c|c|c|c|c|c|}
\hline & \multicolumn{2}{|c|}{$\begin{array}{l}\text { Utiliza Internet para } \\
\text { el negocio }\end{array}$} & \multicolumn{2}{|c|}{$\begin{array}{l}\text { Promociona su } \\
\text { empresa }\end{array}$} & \multicolumn{2}{|c|}{$\begin{array}{c}\text { Se relaciona con } \\
\text { clientes y } \\
\text { proveedores }\end{array}$} & \multicolumn{2}{|c|}{$\begin{array}{l}\text { Vende bienes o } \\
\text { servicios }\end{array}$} \\
\hline & \multicolumn{2}{|c|}{$\mathrm{n}=1205$} & \multicolumn{2}{|c|}{$n=630$} & \multicolumn{2}{|c|}{$\mathrm{n}=630$} & \multicolumn{2}{|c|}{$n=630$} \\
\hline & Prob > & $2=0,000$ & Prob > & $=0,000$ & Prob > & $2=0,000$ & Prob > & $2=0,016$ \\
\hline & $\begin{array}{l}\text { Odds } \\
\text { Ratio }\end{array}$ & $P>Z$ & $\begin{array}{l}\text { Odds } \\
\text { Ratio }\end{array}$ & $P>Z$ & $\begin{array}{l}\text { Odds } \\
\text { Ratio }\end{array}$ & $P>Z$ & $\begin{array}{l}\text { Odds } \\
\text { Ratio }\end{array}$ & $P>Z$ \\
\hline Edad & 0,968 & $0,000^{\star \star \star}$ & 0,957 & $0,000^{\star \star \star}$ & 0,983 & $0,048^{\star \star}$ & 0,980 & $0,002^{\star \star \star}$ \\
\hline Sexo & 0,815 & 0,174 & 0,725 & $0,069^{\star}$ & 1,172 & 0,484 & 0,962 & 0,822 \\
\hline Nivel de estudios & 2,289 & $0,000^{* \star *}$ & 1,078 & 0,197 & 1,267 & $0,003^{* * *}$ & 1,036 & 0,537 \\
\hline Financiamiento & 0,439 & $0,000^{\star \star *}$ & 1,153 & 0,446 & 0,541 & $0,016^{* *}$ & 0,960 & 0,821 \\
\hline Cantidad de socios & 1,511 & $0,003^{\star *}$ & 1,166 & 0,168 & 1,042 & 0,794 & 1,418 & 0,105 \\
\hline Constante & 0,371 & 0,101 & 5,201 & 0,008 & 7,037 & 0,020 & 1,330 & 0,633 \\
\hline
\end{tabular}

Tabla 5: Probabilidad de adopción en micro-emprendedores capacitados en temas seleccionados. ${ }^{* * *}$ Coeficiente significativo, $99 \%$ de confianza. ${ }^{\star *}$ Coeficiente significativo, $95 \%$ de confianza. ${ }^{*}$ Coeficiente significativo, $90 \%$ de confianza.

\begin{tabular}{|l|c|c|c|c|c|c|c|c|}
\cline { 2 - 8 } & \multicolumn{2}{c|}{$\begin{array}{c}\text { Utiliza Internet para el } \\
\text { negocio }\end{array}$} & \multicolumn{2}{c|}{$\begin{array}{c}\text { Promociona su } \\
\text { empresa }\end{array}$} & \multicolumn{2}{c|}{$\begin{array}{c}\text { Se relaciona con } \\
\text { clientes } y \\
\text { proveedores }\end{array}$} & \multicolumn{2}{c|}{$\begin{array}{c}\text { Vende bienes o } \\
\text { servicios }\end{array}$} \\
\cline { 2 - 9 } & \multicolumn{2}{|c|}{$\mathrm{n}=326$} & \multicolumn{2}{c|}{$\mathrm{n}=197$} & \multicolumn{2}{c|}{$\mathrm{n}=197$} & \multicolumn{2}{c|}{$\mathrm{n}=197$} \\
\cline { 2 - 9 } & \multicolumn{2}{c|}{ Prob $>$ chi2 $=0,000$} & Prob $>$ chi2 $=0,0091$ & Prob $>$ chi2 $=0,0016$ & Prob $>$ chi2 $=0,2178$ \\
\cline { 2 - 9 } & Odds Ratio & $\mathrm{P}>\mathrm{z}$ & Odds Ratio & $\mathrm{P}>\mathrm{z}$ & Odds Ratio & $\mathrm{P}>\mathrm{z}$ & Odds Ratio & $\mathrm{P}>\mathrm{z}$ \\
\hline Edad & 0,949 & $0,000^{* * *}$ & 0,979 & 0,116 & 0,989 & 0,536 & 0,986 & 0,275 \\
\hline Sexo & 0,405 & 0,296 & 0,421 & $0,019^{* *}$ & 0,890 & 0,805 & 1,009 & 0,979 \\
\hline Nivel de estudios & 1,754 & $0,000^{* * *}$ & 1,280 & $0,032^{* *}$ & 1,201 & 0,228 & 1,140 & 0,204 \\
\hline Financiamiento & 0,228 & $0,001^{* * *}$ & 1,364 & 0,388 & 0,402 & $0,082^{*}$ & 1,045 & 0,897 \\
\hline Cantidad de socios & 2,331 & $0,016^{* *}$ & 1,446 & 0,114 & 3,740 & $0,030^{* *}$ & 1,390 & 0,118 \\
\hline Constante & 7,338 & 0,146 & 0,706 & 0,780 & 2,915 & 0,553 & 0,421 & 0,451 \\
\hline
\end{tabular}

Se observa que las variables con mayores odds ratios en total de microemprendedores capacitados, son nivel de estudios y cantidad de socios (2,289 y 1,511 respectivamente), esto significa que la razón adoptar internet versus no adoptar internet es 2,289 veces mayor por cada nivel educacional superior y 1,511 veces por cada socio adicional, manteniendo el resto de variables constantes. En sentido contrario, el odds ratio 0,439 asociado a recursos externos para financiamiento de capacitación, implica que la razón de adopción de Internet versus no adopción es 2,278 (1/0,439) veces mayor, en microemprendedores capacitados con recursos internos de la empresa en comparación con microempresarios que utilizan fuentes de financiamiento externas. 
En el grupo de micro-emprendedores que se ha capacitado en administración, marketing y ventas, innovación y TICS, los mayores odds ratios también se asocian a nivel de estudios y cantidad de socios $(2,331$ y 1,754 respectivamente). En forma complementaria, la variable género muestra alta relevancia, el odds ratio 0,405 para género masculino, indica razón de adopción de Internet versus no adopción 2,469 $(1 / 0,405)$ veces mayor en mujeres que en hombres. Adicionalmente, la fuente de financiamiento utilizada para capacitación evidencia aún mayor relevancia en estas temáticas, el odds ratio 0,228 asociado a financiamiento externo, indica proporción 4,386 $(1 / 0,228)$ mayor de adopción de Internet versus no adopción, en grupo de microemprendedores capacitados con recursos propios de la empresa.

\section{DISCUSIÓN}

Los resultados demuestran relación entre capacitación y adopción de Internet y actividades e-Commerce, exceptuando venta de productos. Se plantea como hipótesis, que la venta a través de internet no se vincula con capacitación, pues esta actividad requiere de website habilitado, incorporación de medios de pago como Webpay y desarrollo de procesos asociados a logística de salida para entrega de productos; estos requisitos son independientes al nivel de formación del micro-emprendedor. Considerando las diferencias encontradas por características demográficas, en coherencia con Haider et al. (2015) se propone diseñar programas de capacitación diferenciados, dando prioridad a micro-emprendedores con condiciones asociada a menor uso de Internet y e-Commerce; en términos específicos, se plantea desarrollar programas orientados a microemprendedores hombres mayores de 50 años, que han constituido empresas individuales y presentan nivel de educación menor. El diseño de estos programas considera un mayor número de horas, coaching para mejorar actitud frente a tecnologías y seguimiento en la implementación de tecnologías y e-Commerce en sus empresas. En forma complementaria, se estima la implementación de subsidios focalizados y de apoyo como transporte y alimentación, para incentivar la participación en capacitación de microemprendedores con condiciones desfavorables hacia la adopción de Internet y e-Commerce; también mejorar la supervisión de su asistencia y la finalización de programas de capacitación, por medio de mentores que realicen seguimiento a través de llamados telefónicos o visitas en terreno.

Sobre la alta asociación de financiamiento propio para capacitación con adopción de Internet y e-Commerce, se interpreta que en cursos percibidos como gratuitos, los emprendedores se desempeñan con menor compromiso, pues no perciben potenciales perdidas. En consecuencia, se estima relevante incorporar mayores incentivos o supervisión en programas de capacitación sin cargo al micro-emprendedor, como pago de bonos por finalización, cobertura de financiamiento basado en logro de aprendizajes o firma de carta de compromiso en que se estipule gratuidad del programa a través de financiamiento externo, sólo si se cumple con asistencia a capacitaciones e implementación de acciones en empresa. Las propuestas descritas permitirían fortalecer la participación en capacitación de microemprendedores con condiciones desfavorables y mejorar su desempeño dentro de estos programas, a través de ello, se incrementaría la adopción de Internet y e-Commerce en microempresas en Chile, aumentaría la competitividad de estas organizaciones frente a empresas de mayor tamaño, y se favorecería el crecimiento económico y el empleo (Orjuela, 2006).

\section{CONCLUSIONES}

El objetivo de la investigación es relacionar capacitación de microemprendedores con adopción de Internet y de actividades e-Commerce en sus organizaciones, estimando diferencias por sus condiciones demográficas, número de socios de su empresa y fuente de financiamiento de capacitación. El análisis efectuado evidencia que los micro-emprendedores capacitados son más propensos a uso de Internet, a promocionar productos y a relacionarse con clientes y proveedores a través de Internet, sólo la venta de productos a través de Internet no evidencia esta relación; también se reconocen diferencias entre microempresarios capacitados en gestión, comercialización, ventas, tecnologías de información, innovación, los micro-emprendedores capacitados en estos temas adoptan Internet y promocionan su empresa en mayor medida.

En forma complementaria, los resultados muestran variación por condiciones demográficas, número de socios y fuentes de financiamiento, los micro-emprendedores con menor edad, género femenino, mayor nivel educacional, que integran organizaciones con un mayor número de socios y financian capacitación con recursos de su empresa, adoptan Internet y desarrollan actividades e-Commerce en mayor proporción. De acuerdo con lo señalado, se concluye que la capacitación tiene relación positiva con incorporación de Internet y e-Commerce en microempresas de Chile, y que este vínculo presenta variaciones en función de las condiciones estudiadas.

\section{REFERENCIAS}

Alberti, F., S. Sciascia y A. Poli, Entrepreneurship education: notes on an ongoing debate, In Proceedings of the $14^{\text {th }}$ Annual IntEnt Conference, University of Napoli Federico II, Italy, 4 (7) (2004) 
Afshar Jahanshahi, A., S. X. Zhang y A. Brem, E-commerce for SMEs: empirical insights from three countries, doi: 10.1108/jsbed-03-2012-0039, J. of Small Business and Enterprise Development, 20(4), 849-865 (2013)

Ashourizadeh, S., N. Nasiri y T. Schøtt, Entrepreneurial intention benefitting from education, training and competence: Egypt and Iran, doi: 10.1504/ijesb.2014.065301, International Journal of Entrepreneurship and Small Business, 23(1-2), 94-109 (2014)

Bae, T.J., S. Qian, C. Miao y J.O. Fiet, The relationship between entrepreneurship education and entrepreneurial intentions: A meta-analytic review, doi: 10.1111/etap.12095, Entrepreneurship Theory and Practice, 38(2), 217-254 (2014)

Bruque, S. y J. Moyano, Organisational determinants of information technology adoption and implementation in SMEs: The case of family and cooperative firms, Technovation, 27(5), 241-253 (2007)

CCS, Cámara de Comercio de Santiago, Índice e-commerce 2016, Santiago, Chile (2016)

Cataldo, A. y R. McQueen, IT Alignment in SMEs: Should it be with Strategy or Process? ACIS (2014)

Chong, A.Y.L., A two-staged SEM-neural network approach for understanding and predicting the determinants of $\mathrm{m}$ commerce adoption, doi: 10.1016/j.eswa.2012.08.067, Expert Systems with Applications, 40(4), 1240-1247 (2013)

Colombo, M.G., A. Croce y L. Grillim, ICT services and small businesses' productivity gains: An analysis of the adoption of broadband Internet technology, doi: 10.1016/j.infoecopol.2012.11.001, Information Economics and Policy, 25(3), 171-189 (2013)

Do Paço, A., J.M. Ferreira, M. Raposo, R.G. Rodrigues y A. Dinis, Entrepreneurial intentions: is education enough? doi: 10.1007/s11365-013-0280-5, International Entrepreneurship and Management Journal, 11(1), 57-75 (2015)

Dumas, C., Micro enterprise training for low-income women: The case of the Community Entrepreneurs Programme, doi: 10.1177/097135570101000102, The Journal of Entrepreneurship, 10(1), 17-42 (2001)

Elmuti, D., G. Khoury y O. Omran, Does entrepreneurship education have a role in developing entrepreneurial skills and ventures' effectiveness? Journal of Entrepreneurship Education, 15, 83 (2012)

Gallego, M.D., S. Bueno y J.F. Terreño, Motivations and barriers to set up e-commerce in Spain: A Delphi study, doi; 10.1016/j.estger.2016.08.002, Estudios Gerenciales, 32(140), 221-227 (2016)

Gomezelj Omerzel, D. y B. Antončič, Critical entrepreneur knowledge dimensions for the SME performance, doi:10.1108/02635570810914883, Industrial Management y Data Systems, 108(9), 1182-1199 (2008)

Haider, S.H., M. Asad y A. Aziz, A Survey on the Determinants of Entrepreneurial Training Effectiveness among Micro Finance Institutions of Malaysia, doi: 10.5901/mjss.2015.v6n6s4p396, Mediterranean Journal of Social Sciences, 6(6 S4), 396 (2015)

lansiti, M. y K.R. Lakhani, Digital Ubiquity: how connections, sensors, and data are revolutionizing business (digest summary), Harvard Business Review, 92(11), 91-99 (2014)

Julta, D., P. Bodorik, J. Dhaliwal, Government support for the e-readiness of small and medium sized enterprises, Proceeding of the $35^{\text {th }}$ Annual Hawaii International Conference on System Sciences (2002)

Kessy, S. y S.S. Temu, The impact of training on performance of micro and small enterprises served by microfinance institutions in Tanzania, doi:10.3923/rjbm.2010.103.111, Research Journal of Business Management, 4(2), 103-111 (2010)

Kotelnikov, V., Small and Medium Enterprises and ICT. United Nations Development Programme, Asia-Pacific Development Information Programme (UNDPAPDIP) and Asian and Pacific Training Centre for Information and Communication Technology for Development (APCICT) (2007)

Kotey, B. y C. Folker, Employee training in SMEs: Effect of size and firm type-Family and nonfamily, doi: 10.1111/j.1540-627x.2007.00210.x, Journal of Small Business Management, 45(2), 214-238 (2007)

Lawson, R., C. Alcock, J. Cooper y L. Burgess, Factors affecting adoption of electronic commerce technologies by SMEs: an Australian study, doi: 10.1108/14626000310489727, Journal of small business and enterprise development, 10(3), 265-276 (2003)

Lin, Z., An empirical investigation of user and system recommendations in e-commerce, doi: 10.1016/j.dss.2014.10.003, Decision Support Systems, 68, 111-124 (2014) 
Mandakovic, V., A. Abarca Montero y J. E. Amorós, Reporte Nacional de Chile 2015, Global Entrepreneurship Monitor (2015)

Martin, B.C., J.J. McNally y M.J. Kay, Examining the formation of human capital in entrepreneurship: A metaanalysis of entrepreneurship education outcomes, doi: 10.1016/j.jbusvent.2012.03.002, J. of Business Venturing, 28(2), 211-224 (2013)

Millan, J. M., E. Congregado, C. Roman, Van Praag, M. y A. Van Stel, The value of an educated population for an individual's entrepreneurship success, doi: 10.1016/j.jbusvent.2013.09.003, Journal of business venturing, 29(5), 612-632 (2014)

Ministerio de Economía, Fomento y Turismo de Chile. Cuarta encuesta de microemprendimiento (EME4), https://goo.gl/ntg9Du. Acceso: 10 de Enero (2017), Santiago, Chile (2016)

Morgan Stanley Capital International, MSCI World Index Stock 2016, https://goo.gl/76HMvF. Acceso: 08 de Enero (2017)

Nasco, S.A., E.G. Toledo y P.P. Mykytyn, Predicting electronic commerce adoption in Chilean SMEs, doi: 10.1016/j.jbusres.2007.06.047, Journal of Business Research, 61(6), 697-705 (2008)

Nasri, N. y Y. P. Iskandar, Pedagogy paradigm: Competency in e-commerce training for entrepreneurs. In Technology, Informatics, doi: 10.1109/time-e.2015.7389752, Management, Engineering y Environment (TIME-E), 2015 International Conference on (pp. 82-86), IEEE (2015)

Njoroge, C.W. y J.M. Gathungu, The Effect of Entrepreneurial Education and Training on Development of Small and Medium Size Enterprises in Githunguri District-Kenya, International Journal of Education and Research, 1(8), 1-22 (2013)

Ollivier Fierro, J., El problema del rezago tecnológico de las microempresas industriales y alternativas de solución, Contaduría y administración, (221), 137-160 (2007)

Orjuela, F.N., Elementos para el estudio de la microempresa latinoamericana. Latinoamérica, Revista de Estudios Latinoamericanos, (43), 153-174 (2006)

Petridou, E., A. Sarri y L. P. Kyrgidou, Entrepreneurship education in higher educational institutions: the gender dimension, doi: 10.1108/17542410910961569, Gender in Management: An Inter. Journal, 24(4), 286-309 (2009)

Putta, S.S., Improving Entrepreneur's Management Skills through Entrepreneurship Training, doi: 10.5958/0976-478x.2014.00334.6, Journal of Commerce and Management Thought, 5(3), 459 (2014)

Qureshi, S., How does information technology effect development? Integrating theory and practice into a process model, National Bureau of Economic Research (2005)

Ramírez-Pérez, H.X., A.M. E. Smith, J. E. Franco-López, J. Román-Maqueira y E. S. Morote, Effects of training method and age on employability skills of mexican youth entrepreneurs, Journal of Entrepreneurship Education, 18(2), 125 (2015)

Rauth Bhardwaj, B., Impact of education and training on performance of women entrepreneurs: A study in emerging market context, doi: 10.1108/jeee-05-2013-0014, Journal of Entrepreneurship in Emerging Economies, 6(1), 38-52 (2014)

Simmons, G.J., G. A. Armstrong y M. G. Durkin, A conceptualization of the determinants of small business website adoption: setting the research agenda, doi:10.1177/0266242608088743, International Small Business Journal, 26 (3), 351-389 (2008)

Subsecretaría de Telecomunicaciones de Chile, Series de conexiones de Internet fija, https://goo.gl/sKxafH. Acceso: 04 de Marzo (2017), Santiago, Chile (2016)

Turban, E., D. King, J. K. Lee, T.P. Liang y D. C. Turban, Electronic commerce: A managerial and social networks perspective, doi:10.1007/978-3-319-10091-3, Springer Texts in Business and Economics (2015)

Valerio, A., B. Parton y A. Robb, Entrepreneurship education and training programs around the world: dimensions for success, doi: 10.1596/978-1-4648-0202-7, World Bank Publications (2014)

Vidal, C. L., L.L. López, J. A. Rojas y M.M. Castro, Desarrollo de Sistema Web de Reclutamiento y Selección y de Directivos por Competencias mediante PHP Codelgniter 3.0, Infor. Tecnológica, 28(2), 203-212 (2017)

Wolcott, P., M. Kamal y S. Qureshi, Meeting the challenges of ICT adoption by micro-enterprises, doi: 10.1108/17410390810911212, Journal of Enterprise Information Management, 2, 94(2), 532-551 (2008) 
